\title{
La destrucción de Jericó a fines de la Edad del Bronce Antiguo III: un análisis preliminar de diferentes interpretaciones
}

Q Katherine Kifer

Universidad de Buenos Aires, Argentina

Fecha de recepción: 25 de julio de 2021. Fecha de aceptación: 18 de agosto de 2021.

\begin{abstract}
Resumen
El objetivo del presente artículo es analizar las perspectivas sobre las transformaciones ocurridas en Jericó a fines de la Edad del Bronce Antiguo III ( $c a$. 2700/2650-2300 a.C.). Para esto se comparan brevemente los restos materiales correspondientes al Bronce Antiguo III y el período siguiente, el Bronce Antiguo IV (ca. 2300-1950 a.C.). Tras ello, se pretende debatir sobre si estos cambios deben interpretarse como un "colapso" del sistema o una "adaptación paulatina" a las nuevas condiciones y demandas regionales. Al respecto, cabe señalar que mientras muchos sitios eran abandonados, Jericó experimentó un tiempo de florecimiento económico y desarrollo urbano sin precedentes, pero que en torno al 2350 a.C. se vio interrumpido de manera abrupta: al parecer, ingresaron enemigos a la ciudad, saqueando todo lo que allí había, incluidas las riquezas del palacio y el templo. En simultáneo, es probable que incendiaran las murallas de la ciudad. Años después, el asentamiento sería rehabitado por grupos de pastores-guerreros procedentes, aparentemente, de la Alta Mesopotamia, quizás motivados a trasladarse por causa de las alteraciones medioambientales en sus tierras de origen.
\end{abstract}

Palabras clave: Jericó, Bronce Antiguo, desurbanización, colapso

The Destruction of Jericho at the End of the Early Bronze III: A Preliminary Analysis of Different Interpretations

\footnotetext{
Abstract

The aim of the present article is to analyze some perspectives on the transformations that took place in Jericho at the end of the Early Bronze Age III (ca. 2700$2300 \mathrm{BCE}$ ). Therefore, a brief comparison will be made between the materials
} 
that belonged to the Early Bronze Age III and to the following period, the Early Bronze Age IV (ca. 2300-1950 BCE). Afterwards, a discussion will be held to decide whether the changes should be interpreted as a system collapse or as a gradual adaptation of the new conditions and regional demands. In this regard, and although many areas were abandoned, Jericho experienced a flourishing period and urban development without precedent, but towards 2350 BCE such changes were abruptly interrupted: enemies entered and looted everything present there, including the jewelry of the palace and the temple. Simultaneously, the walls of the city were set on fire. Years later, the settlement was inhabited by shepherd-warrior groups coming mainly from Upper Mesopotamia, likely motivated by environmental issues.

Keywords: Jericho, Early Bronze Age, desurbanization, collapse

\section{Introducción}

El sitio de Tell es-Sultán, donde estaba ubicada la ciudad antigua de Jericó, fue excavado en distintas campañas a fines del siglo XIX y comienzos del XX: por Charles Warren entre 1867 y 1868, por Ernst Sellin y Carl Watzinger entre 1907 y 1909, y por John Garstang, entre 1930 y 1936. A mediados del siglo pasado, entre 1952 y 1956, la arqueóloga británica Kathleen Kenyon realizó una serie de campañas famosas cuando, aplicando novedosos métodos arqueológicos, ayudó a establecer la cronología del valle del Jordán y, por extensión, de todo el Levante meridional, desde el período Neolítico hasta la Edad del Bronce (Kenyon, 1966 [1957]; Davis, 2008: 101-154). En 1997, el Departamento de Antigüedades y Patrimonio Cultural de Palestina, en cooperación con la Universidad de Roma, inició un nuevo proyecto de exploración en la región, el cual continúa hasta la actualidad (Nigro, 2014).

El asentamiento de Jericó estaba situado sobre una llanura fértil en el margen occidental del río Jordán; debía su existencia al arroyo que fluía a sus pies, cuya fuente era posiblemente algún depósito subterráneo alimentado por las lluvias de las tierras altas de Judea. El venero de Ain el-Sultán hacía verdaderamente productivo el rico terreno del valle. Su estratégica ubicación la convirtió en un influyente centro urbano en relación con los intercambios.

El objetivo de este trabajo es analizar el final abrupto de la primera fase de urbanización del asentamiento, cuyo auge corresponde a la fase arqueológica Sultan IIIc, datada en la Edad del Bronce Antiguo III (ca. 2700-2300 a.C.) $)^{1}$ (Nigro et al., 2019: 4). Luego de un breve abandono, el sitio volvió a estar ocupado, pero esta vez por pastores con una fuerte impronta guerrera, quienes durante todo Sultan IIId decidieron no reconstruir la ciudad. Esta última fase coincide con la Edad del Bronce Antiguo IV (ca. 2300-2000 a.C.), la cual también recibe el nombre de Bronce Medio I, de Intermedio Bronce Antiguo-Bronce Medio o simplemente Bronce Intermedio (Greenberg, 2019: 137-139).

1 Conviene destacar que la cronología de estos períodos sigue en debate. Mientras que la mayor parte de los fechados coinciden en finalizar el Bronce Antiguo III en torno al 2400 a.C., en el caso de Jericó parece que este período dura un siglo más (Nigro et al., 2019). 


\begin{tabular}{lcc}
\hline Fases arqueológicas & Edad del Bronce Antiguo (BA) & Fechas aproximadas \\
\hline Sultan III a & BA I & $3500-3000$ a.C. \\
\hline Sultan III b & BA II & $3000-2700$ a.C. \\
\hline Sultan III c & BA III & $2700-2300$ a.C. \\
\hline Sultan III d & BA IV & $2300-2000$ a.C. \\
\hline
\end{tabular}

En primer lugar, realizaremos una breve descripción de los restos materiales hallados durante las distintas excavaciones dirigidas por los arqueólogos Kathleen Kenyon y Lorenzo Nigro en Jericó, correspondientes al Bronce Antiguo III y IV. En segundo lugar, sintetizaremos las diferentes interpretaciones ofrecidas por los investigadores para explicar estos cambios. Finalmente, propondremos nuestra perspectiva sobre lo sucedido, insistiendo en la necesidad de repensar, a la luz de los nuevos estudios, algunas de las hipótesis que abordaron las transformaciones ocurridas en aquella ciudad a finales del Bronce Antiguo III.

\section{Jericó en la Edad del Bronce Antiguo III}

A partir del 2700 a.C., Jericó consolida su urbanización, destacando la construcción de un palacio y una nueva muralla, más gruesa y resistente. En la parte más alta del montículo se ubicaba un palacio de tres alas y varias salas. En su interior se hallaron instalaciones industriales para la fabricación de alimentos y diferentes tipos de objetos, entre ellos, una daga con mango de cuero, una cabeza de maza de mármol egipcio, una jarra roja pulida, una cabeza de toro de marfil, un hacha de cobre, vasos ceremoniales, piedras de molienda, morteros, cuchillas de pedernal e inclusive, un disco delgado de basalto utilizado como torno de alfarero (Nigro, 2019a: 91-94).

La existencia de un palacio -cual residencia de la élite-sugiere que el poder se había concentrado en pocas manos (de Miroschedji, 2019: 164). Con relación a su ubicación, podemos suponer que ofrecía un buen panorama desde el cual observar el oasis, el cotidiano quehacer de los habitantes del sitio y el ingreso o salida de gente por las puertas de la ciudad. Su ubicación le permitía tener control sobre el manantial, las zonas de cultivo y un rápido acceso a las rutas de intercambio.

Por lo que se refiere al sistema de fortificación, la ciudad contaba con una primera muralla de $4 \mathrm{~m}$ de ancho y $8 \mathrm{~m}$ de alto, a lo que se sumaba un muro exterior de 1,5 m de ancho (Nigro, 2019a: 85-90). Ambas estructuras habían sido construidas con ladrillos de adobe sobre cimientos de piedra y estaban conectadas por muros transversales. Además, el muro interior estaba reforzado con vigas de madera. En relación con la construcción de la nueva muralla, Gallo (2013) ha planteado que dicho proyecto requería de una organización socioeconómica articulada, un cuerpo social jerárquico, la capacidad de reunir grandes cantidades de materiales de construcción y la necesidad de proteger el núcleo de asentamiento y bienes de la ciudad de ataques externos. 
Un conjunto de viviendas fue identificado en la sección norte de la ciudad. Estaban construidas con ladrillos de adobe, madera y piedras; en su interior se hallaron herramientas de pedernal, un sello cilíndrico de hueso, una balanza para pesar metales e inclusive, cabezas de maza egipcias y artefactos cerámicos (Nigro, 2020: 190). Afortunadamente, parte de las vasijas contenían residuos orgánicos. Tras ser analizados, se descubrió que la dieta de la población de Jericó estaba basaba en el consumo de frutos -como la oliva-, vegetales, legumbres, ovinos, caprinos, bovinos, e incluso pescados, gacelas, hipopótamos, onagros y jabalíes (Nigro, 2019a: 95). Algo semejante ocurría con otros centros urbanos del Levante, donde además de los productos de la campaña rural se hallaron restos de animales exóticos -salvajes- o de estatus -cerdos y otro tipo de ganado- jóvenes o subadultos (Gaastra et al., 2020: 16).

La abundancia de productos, los diversos tipos cerámicos -ya sean oriundos de Khirbet Kerak, Biblos, Sidón o de imitación local-y la presencia de paletas, sellos, balanzas, vasos o cabezas de maza egipcias, demostraron que Jericó era un punto de convergencia para intercambios a larga distancia (Nigro, 2019a: 97; 2019b: 144). A nivel local también intercambiaban aceites, vinos, sal, maderas, azufre, betún del mar Muerto, caracolas del Mediterráneo y el mar Rojo, maderas, cobre -de Wadi Feynan y Sinaí- y marfiles tallados (Nigro, 2019a: 97-98). Dichos contactos no sólo brindaban la posibilidad de obtener productos exóticos y/o nuevos conocimientos especializados sino también abrían la posibilidad de establecer alianzas con las élites de otras urbanizaciones, asentamientos rurales e inclusive con Egipto (Sala, 2012: 287). Dependiendo la ocasión las relaciones con este último podían ser comerciales o diplomáticas. De donde resulta que el poder político central controlaba la producción, la distribución y los intercambios o trueques de bienes (Milevski, 2016: 243).

Por último, es menester destacar que los habitantes de Jericó depositaban los huesos de sus fallecidos en enterramientos múltiples en los cuales también se depositaban vasijas cerámicas (Kenyon, 1960: 121).

\section{Incendio y destrucción}

Alrededor del 2350 a.C. hallamos evidencias que sugieren que tanto el palacio como las murallas sufrieron un violento ataque (Nigro, 2019a: 103; 2020: 194). Lo suponemos porque los ladrillos de adobe de las murallas presentaban un color rojo brillante y a sus costados permanecían restos de cenizas. Al tiempo que ambas murallas entraban en combustión y junto a éstas, las viviendas colindantes, el mobiliario, las columnas y riquezas del palacio parecen haber sido saqueadas. Todas las viviendas halladas en el interior del sitio estaban cubiertas de una capa de cenizas y sus ladrillos carbonizados y rotos.

Considerando que los bienes del palacio - columnas y mobiliario- fueron quitados previo al incendio, Nigro (2020: 194) sugiere que el incendio fue intencional y se debió a un ataque externo. Como resultado el techo del palacio se derrumbó y aplastó todo lo que permanecía debajo. Una situación similar parece haber ocurrido en el palacio de Khirbet al-Batrawy y el de Ebla. Ambos sufrieron una 
violenta conflagración; fueron asaltados e incendiados. La cantidad de objetos abandonados in situ llevó a pensar que los ataques fueron repentinos y causantes de las destrucciones (Nigro, 2017: 164; Vega Ruiz, 2019: 91-92). En rasgos generales, el uso del fuego -incendio del asentamiento- para triunfar sobre el enemigo no sólo tenía como objetivo erradicarlo del espacio sino también cercenar su identidad, su pasado y su prestigio (Di Bennardis, 2014: 45).

\section{Jericó en la Edad del Bronce Antiguo IV}

Por un corto tiempo, Jericó permaneció deshabitado. Recién alrededor del año 2300 a.C. comenzaron a aparecer asentamientos semipermanentes en el tell y las colinas adyacentes (Nigro, 2021: 282). Años más tarde, construyeron viviendas de adobe. En opinión de Nigro (2019b: 145), los nuevos pobladores eran seminómadas que habitaban el oasis durante el verano, practicaban la trashumancia en otoño y primavera y contaban con conocimientos vinculados a la industria del bronce.

Los diferentes tipos de enterramientos hallados en el lugar sugieren que, a pesar de compartir un estilo de vida móvil, los recién llegados no conformaban un grupo homogéneo. Kenyon (1966 [1957]: 97-107) halló alrededor de 360 tumbas que clasificó de la siguiente manera:

» Tumba-daga: construidas con mucho cuidado y precisión, consistían en un pozo vertical poco profundo, en cuyo fondo se abría paso una cámara pequeña cerrada con una sola puerta, donde se depositaban uno o dos difuntos, de distintas edades y sexo, acompañados por ofrendas sencillas y austeras, las más de las veces sólo por una daga.

» Tumbas-cerámica: a diferencia de las anteriores, aquí el ajuar está compuesto por piezas de alfarería y las cámaras son más amplias, aunque toscamente ejecutadas; los restos humanos corresponden en su mayoría a un solo individuo por cámara, siempre un adulto, cuyo cuerpo se colocaba desmembrado una vez que la carne estaba ya descompuesta.

» Tumbas de pozo cuadrado: en este caso, el pozo que conducía a la cámara tenía planta rectangular y el ajuar combinaba vasijas y dagas, además de jabalinas ocasionales.

» Tumbas tamaño mayor: las cámaras eran mucho más espaciosas que los casos anteriores, pero contenían tan sólo un individuo acompañado de varias armas y vasijas. Algunas presentaban grafitis de arbustos y animales de largos cuernos en sus paredes.

" Tumbas-cuenta o miskin: parecidas al tipo tumbas-cerámica, pero más pequeñas y con muy pocos objetos en su interior, sólo unas pocas cuentas y algunos clavos de adorno.

Según Greenberg (2019: 164), la gran cantidad de tumbas que fueron halladas en todo el Levante meridional pueden organizarse en un continuo con dos polos opuestos: tumbas individuales y tumbas comunales. Desde este punto de vista, la mayoría de las tumbas de Jericó datadas en el Bronce Antiguo IV corresponderían a la categoría de tumbas individuales. De manera interesante, el hecho de que cada difunto haya sido enterrado por separado parece relegar la importancia del 
parentesco a favor de la comunidad, sin negar la individualidad de cada uno de sus integrantes. En función de los estudios realizados sobre la procedencia de dichos metales, Greenberg (2019: 167) argumenta que las armas depositadas en las tumbas habían sido elaboradas en el norte del Levante. De ahí que podrían haber sido trasladadas por los mismos pastores o bien éstos habrían obtenido dichos materiales, tiempo después, mediante relaciones de intercambio (Massa y Palmisano, 2018: 82; Nigro et al., 2008: 206; Greenberg, 2019: 166-168).
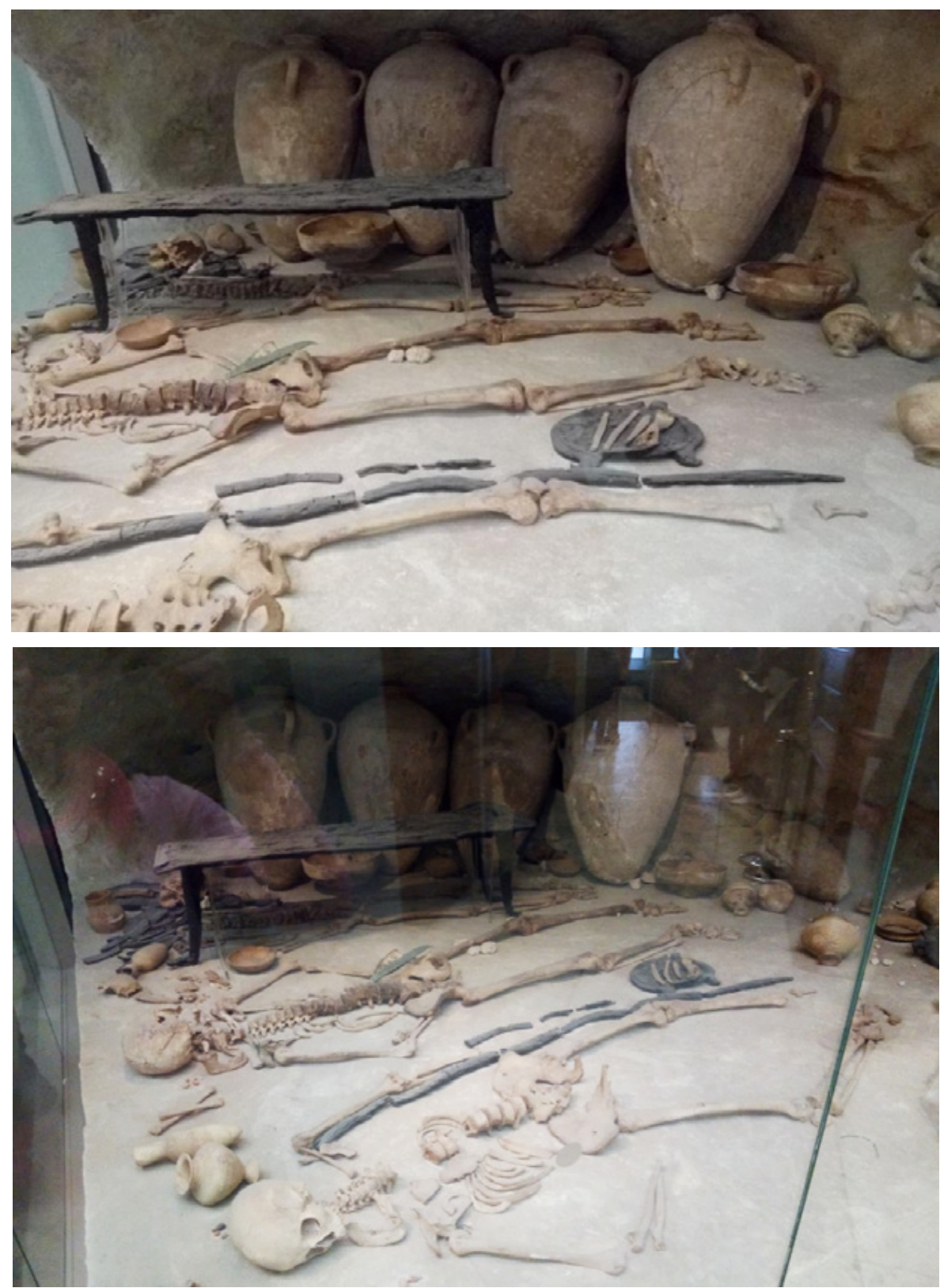

Fig. 1 y 2. Reconstrucción de la tumba P19 correspondiente a la Edad del Bronce Antiguo IV, Museo Británico, 2020. Fotografiado por Katherine Kifer.

Sobre las ruinas de la ciudad y la colina adyacente se hallaron restos de cerámicas que no mantenían semejanza con las del Bronce Antiguo III. En general, 
eran de color gris, verdoso, amarillento o rosado; no presentaban un acabado pulimentado sino una textura áspera, de cocción deficiente y paredes delgadas. Los recipientes estaban hechos a mano. Los cuellos de las vasijas -generalmente altos- habrían sido adheridos a los cuerpos hechos a mano mediante una rueda de alfarero relativamente rápida. Su decoración eran simples líneas onduladas (Kenyon, 1966 [1957]: 94).

En resumen, la falta de continuidad entre estilos cerámicos de ambos períodos, la ausencia de arquitectura monumental, el modelo de asentamiento sin un ordenamiento jerárquico visible y la existencia de una economía basada principalmente en la cría de ovejas podría indicar el predominio del pastoralismo y una tendencia a la ruralización durante estos años.

\begin{tabular}{|c|c|c|c|}
\hline PERÍODO & Sultan Illc & \multirow{4}{*}{$\begin{array}{l}\text { l } \\
\mathrm{n} \\
\mathrm{c} \\
\mathrm{e} \\
\mathrm{n} \\
\mathrm{d} \\
\mathrm{i} \\
\mathrm{o}\end{array}$} & Sultan IIld \\
\hline Cerámica & $\begin{array}{l}\text { Cerámicas pulimentadas. } \\
\text { Producción local y de } \\
\text { importación como la } \\
\text { cerámica Khirbet Kerak. }\end{array}$ & & $\begin{array}{l}\text { Ausencia de cerámicas } \\
\text { pulimentadas: textura áspera } \\
\text { y frágil. } \\
\text { Producción local. }\end{array}$ \\
\hline Arquitectura & $\begin{array}{l}\text { Construcción de un } \\
\text { palacio y una doble } \\
\text { muralla con ladrillos de } \\
\text { adobe, piedras y madera. }\end{array}$ & & $\begin{array}{l}\text { Viviendas construidas con } \\
\text { ladrillos de adobe color } \\
\text { verdoso. } \\
\text { No hay indicios de } \\
\text { reconstrucción de la muralla. }\end{array}$ \\
\hline Tumbas & Enterramientos múltiples. & & $\begin{array}{l}\text { Enterramientos individuales: } \\
\text { - Tumba-daga. } \\
\text { - Tumbas-cerámica. } \\
\text { - Tumbas pozo-cuadrado. } \\
\text { - Tumbas tamaño mayor. } \\
\text { - Tumbas-cuenta o miskin. }\end{array}$ \\
\hline
\end{tabular}

\section{Perspectivas interpretativas}

A continuación, presentamos brevemente algunas de las hipótesis que intentan explicar qué fue lo que ocurrió en el sur del Levante a finales del Bronce Antiguo III. En líneas generales, podríamos señalar dos corrientes: por un lado, aquella que afirma que hubo una ruptura, es decir, un cambio rotundo entre la sociedad de este período y la del posterior -el Bronce Antiguo IV- y, por otro lado, la que asegura que se trató de una adaptación a un contexto cambiante en el cual las sociedades optaron por modificar sus modos de vida.

\section{Rupturistas}

\section{Hipótesis de la migración amorrea}

Desde un comienzo, los cambios entre el Bronce Antiguo III y IV fueron interpretados como producto de una invasión y/o migración de poblaciones foráneas (por ej., Albright, 1961; Lapp, 1966: 82-116). Si bien se 
postularon distintos posibles orígenes, la hipótesis que obtuvo mayor apoyo fue aquella que sostenía que se trataba de poblaciones amorreas. Entre sus principales referentes se encontraban William F. Albright y Kathleen Kenyon, esta última basando gran parte de su argumento justamente en la evidencia hallada en Jericó. A su entender, los responsables de los trastornos ocurridos habían sido los amorreos, semitas occidentales procedentes de los bordes semiáridos del Creciente Fértil, quienes realizaron incursiones y conquistaron partes de Palestina, de Siria y de Mesopotamia (por ej., Kenyon, 1960: 133-159).

\section{Hipótesis del cambio climático}

En 1993, Harvey Weiss y colegas sostuvieron que un fenómeno climático había provocado la crisis del Imperio acadio, el fin del Reino Antiguo egipcio y el declive de las primeras urbanizaciones en Siria y Palestina (Weiss et al., 1993). Estudios paleoclimáticos recientes ratificaron la existencia de este cambio abrupto, el cual denominaron como "evento árido de 4,2 cal kyr BP", datado aproximadamente entre 2250 y 1950 a.C. (Höflmayer, 2017). Durante este período se observó un aumento de las temperaturas, cierta disminución de las precipitaciones y un cambio en la aridez del suelo. En simultáneo, el abandono generalizado de las tierras agrícolas del norte de Mesopotamia como importantes movimientos poblacionales dirigidos hacia el Levante meridional y el sur de Mesopotamia (Kaniewski et al., 2008; 2019; Riehl, 2008). De haber sido así, seguramente los habitantes de las estepas semiáridas habrían sido las primeras víctimas de la sequía.

\section{Hipótesis del colapso}

Contra la hipótesis de la migración/invasión, William G. Dever había propuesto la idea de un colapso que se debía más bien a factores internos, donde las poblaciones sedentarias dependientes de las ciudades se volcaron hacia una economía pastoril, de ahí la menor cantidad de asentamientos urbanos y la mayor dispersión de las aldeas (por ej., Dever, 1980; 1992). Esta idea del cambio endógeno ganó adeptos, considerando este fenómeno como parte de una serie de ciclos de urbanización y desurbanización que atravesó la región durante toda la Edad del Bronce y del Hierro (por ej., Finkelstein, 1996).

La hipótesis del cambio climático fue incorporada dentro de este modelo, pues se habría tratado de un factor que terminaba de actuar sobre tendencias locales hacia la desurbanización. Por ejemplo, Pierre de Miroschedji (2009) también asegura que a fines del Bronce Antiguo III ocurrió un colapso general caracterizado por el desplazamiento de las condiciones de vida de grupos sedentarios hacia el nomadismo. El "colapso" lo considera una consecuencia de la acumulación de eventos locales con estrecha relación con la interrupción de redes de intercambios, cambios ambientales que provocaron sequías generalizadas, el arribo de grupos amorreos o la disminución de los contactos comerciales con Egipto. En este sentido, dicho colapso había sido el destino de un proceso de involución de larga duración que había comenzado a finales del Bronce Antiguo II (de Miroschedji, 2009: 116, 119). 


\section{Continuistas}

En cierto sentido, muchos de quienes hablan de colapso podrían ser considerados también como continuistas, pues sostienen la adaptación de estas poblaciones al nuevo contexto. Sin embargo, conviene considerarlos por separado, pues resaltan el aspecto gradual del proceso antes que las rupturas.

Su crítica a la hipótesis de la migración amorrea se basa en que, si bien no se puede descartar algún movimiento poblacional, la escala habría sido bastante pequeña y en todo caso actuaría sobre tendencias previas hacia la desurbanización. En efecto, los fechados más recientes ubican el final del Bronce Antiguo III en todo el Levante meridional en torno al 2500 a.C., lo que también pone en duda el peso de la hipótesis del cambio climático pues, como recuerda J. David Schloen (2017), estas modificaciones habrían ocurrido dos o tres centurias después de la desaparición de muchas ciudades. Este mismo autor sostiene, además, que la noción de "colapso" no sería aplicable para el sur del Levante en tanto la transición no debería definirse como una desintegración económica sino, más bien, como una reorientación hacia el pastoreo con el fin de alcanzar una mayor integración económica pan-levantina cuyo eje se encontraba en Siria, específicamente en Ebla y sus asentamientos satélites. En este sentido, las élites sureñas habrían "optado" por abandonar el antiguo sistema agrícola en pos de promover el pastoralismo -pastoreo, producción o transporte de lana- convirtiendo los centros urbanos en parte de una economía lanera más amplia en tanto esto les brindaría mayores riquezas, que incluían la obtención de joyas o armas de bronce. En pocas palabras, las nuevas oportunidades económicas en el norte habrían hecho obsoleto el antiguo modelo urbano (Schloen, 2017: 63).

Raphael Greenberg $(2017$; 2019) ofrece una explicación similar: la desaparición de los centros urbanos no debería entenderse como un "colapso" en tanto desintegración sino como reestructuración asociada a cambios globales, un período de negociación y experimentación. El aumento de la demanda lanera en Siria habría despertado el interés tanto de ciertas comunidades sedentarias como agropastoriles quienes habrían optado por abandonar sus antiguos modos de vida para dedicarse a la gestión de animales hacia Ebla. En este sentido, el Bronce Antiguo IV debería interpretarse como el punto de llegada de un proceso largo -de éxodo/migración- iniciado en el final del Bronce Antiguo II (Greenberg, 2017: 48; 2019: 125-128).

De igual manera, Schwartz (2017) considera que, si las instituciones de determinados sitios sufrían transformaciones, los asentamientos asociados a éstos también podían sufrir cambios e inclusive terminar siendo abandonados. No obstante, el abandono de los asentamientos no estaba sincronizado (Schwartz, 2017: 120). Por su parte, Felix Höflmayer (2017) sostiene que los colapsos de las urbanizaciones nunca son completos, sino que negocian entre dos polos: el de la continuidad y el de la disrupción, entre el estancamiento y la revolución. En su opinión, el sistema urbano declinó paulatinamente (Höflmayer, 2017: 2, 19). 


\section{Discusión: hacia una perspectiva alternativa}

Las diferentes posturas analizadas intentan explicar los factores que provocaron la crisis a finales del Bronce Antiguo III en el Levante meridional. Resta saber ahora, en función de la evidencia descrita en secciones anteriores, su aplicabilidad al caso específico de Jericó.

Es evidente que la cantidad de centros urbanos disminuyó (Milevski, 2010: 176); muchas ciudades fueron abandonadas o experimentaron profundas transformaciones en aquellos años, ya sea porque sus pobladores decidían emigrar en busca de mejores oportunidades o porque sus gobernantes optaban por incentivar -en mayor nivel- el pastoralismo en pos de integrarse a una economía lanera más amplia, pero puede ser que no haya ocurrido así en todos los asentamientos del sur del Levante. Jericó y otros sitios, como por ejemplo Khirbet al-Batrawy, continuaron siendo centros urbanos fortificados cuyas comunidades practicaban la agricultura y reprodujeron un modelo de organización político-social centralizada por varias décadas más. En Khirbet al-Batrawy también fue hallado un palacio en cuyo interior había diferentes tipos de objetos, entre ellos, vasos rituales, ánforas y hachas de cobre (Nigro, 2016: 159, 163).

Como dijimos, Jericó habría experimentado un período de crecimiento agrícola y desarrollo urbano nunca visto, posiblemente beneficiado por una menor competencia de otros centros, lo que le habría facilitado acceder a las rutas de intercambio. No obstante, de ser así, esto podría haberlo expuesto a un ataque de pastores-guerreros, ante lo cual ya no contaría con la posibilidad de establecer alianzas con otras ciudades cercanas. En otras palabras, su relativo aislamiento, si bien lo beneficiaba económicamente, lo exponía a sufrir una estrepitosa caída.

A nuestro modo de ver, lo más probable es que haya habido un cambio abrupto en la composición social de quienes vivían en el tell, como indica el abandono del sitio tras la destrucción y la reocupación, décadas después, con estructuras y una cultura material completamente distintas. Estos nuevos habitantes estarían vinculados a una cultura guerrera, como indican las armas en los ajuares funerarios. Los habitantes de Jericó en el Bronce Antiguo III, si bien contaban con extensas murallas, quizás no estaban tan habituados a la guerra como sus sucesores. $^{2}$ Si estos últimos fueron quienes incendiaron y destruyeron los edificios, entonces debemos concluir que no parecían interesados en las alianzas pacíficas, sino que su estrategia consistía en la aniquilación de sus enemigos y el saqueo de sus bienes.

Por supuesto, es difícil establecer el origen de estos pastores-guerreros. Si su economía estaba orientada hacia Siria, como sugieren varios autores, entonces cabe asumir que podrían haber tenido vínculos -alianzas político-económicascon poblaciones semíticas del norte occidental como con otras comunidades y/o

2 Nigro (2020: 192) sugiere, a partir de un hacha y una daga halladas en el interior del palacio del Bronce Antiguo III, que en esta ciudad pudo haber comenzado a emerger un incipiente grupo guerrero. 
ciudades más lejanas -pongamos el caso de Ebla o sus asentamientos satélites- a quienes habrían provisto de animales o productos derivados -lana, lácteos- a cambio de objetos lujosos, cerámicas o armas de bronce.

Su traslado hacia el sur del Levante, vía el corredor del valle del Jordán, quizás estuvo motivado por las alteraciones medioambientales en sus tierras de origen. $\mathrm{Al}$ respecto, conviene señalar que la destrucción está datada aproximadamente en 2350 a.C. mientras que el "evento árido de 4,2 cal kyr BP", habría comenzado a partir de 2250 a.C. Si bien todavía tenemos un siglo de diferencia entre uno y otro, es legítimo preguntarse si los efectos de este cambio ya habrían comenzado a actuar primero en las regiones semiáridas, lo que obligó entonces a poblaciones marginales a dirigirse hacia zonas con pasturas adecuadas para el pastoreo, como era la fértil llanura del Jordán.

Vemos, entonces, que las perspectivas propuestas hasta ahora, si bien sirven para explicar los cambios a escala de todo el Levante meridional, resultan insuficientes para comprender lo que sucedió en Jericó. Aquí, en cambio, puede ser que se hayan combinado distintos elementos, lo que daría lugar a una excepción que, de todas maneras, confirmaría aspectos de todas las propuestas. En síntesis, es posible que las tendencias regresivas comunes que conducían a una paulatina desurbanización, en la cual algunos centros urbanos se reorientaron al pastoralismo, hayan sido aprovechadas por las poblaciones de Jericó para desarrollar sus actividades agrícolas a la par del mejoramiento del sistema urbano vigente -a partir de la construcción de un palacio y murallas más gruesas y resistentes- y del fortalecimiento del poder de la élite. No obstante, el escenario global ya se había transformado y ante el ataque de grupos enemigos, los habitantes de Jericó no pudieron hacer frente, dejando a la ciudad expuesta al saqueo y posterior incendio de todo su interior, un verdadero colapso. Enseguida la ciudad fue abandonada y reocupada tiempo después por poblaciones pastoriles, posiblemente semitas occidentales vinculadas a otras poblaciones móviles del Levante septentrional.

\section{Consideraciones finales}

Las evidencias halladas en el sitio de Jericó constatan los fuertes cambios que atravesaron las poblaciones del Levante meridional en el paso del Bronce Antiguo III al IV, caracterizados por una marcada desurbanización que contrasta con la situación predominante siglos antes. Los indicios de incendio y de destrucción nos hacen pensar que la dinámica del proceso habría conllevado un verdadero colapso. De manera semejante, el rotundo cambio de la cultura material entre un período y otro permite suponer la presencia de nuevas poblaciones con otras formas de vida y de identidad cultural. En este sentido, si bien es posible que toda la región se haya orientado paulatinamente hacia el pastoralismo y/o a la demanda lanera del norte del Levante, no creemos que éste haya sido el caso de Jericó quien estaba cada vez más abocada al desarrollo agrícola, al fortalecimiento del sistema urbano y a la promoción de los intercambios de corta y larga distancia. De todas formas, esta decisión habría conducido a un relativo aislamiento, quedando expuesta a los ataques de pastores que no compartían los ideales urbanos de la élite gobernante en Jericó. 
Por supuesto, nuestra reconstrucción no es más que una conjetura, pero considera la posibilidad de que, más allá de las tendencias comunes, no todas las personas, y en efecto las ciudades y/o asentamientos, hayan respondido de la misma manera a las nuevas oportunidades, sino que, mientras unas decidían involucrarse en la economía pastoril otras preferían reforzar los modos de vida establecidos. Asumir que la acción de los grupos sociales se explica por la aplicación de un modelo teórico u otro creemos que simplifica la complejidad de la realidad social. A nuestro modo de ver, la élite de Jericó habría apostado por una estrategia que se vio interrumpida -de manera rotunda- ante un ataque enemigo al que no pudieron hacer frente. El restablecimiento de una organización social urbana se tornó inasequible.

A medida que aumenten el número de hallazgos y los resultados de los nuevos estudios, estamos seguros de que podremos precisar más nuestra propuesta, confirmando la relevancia de algunos factores y descartando otros, para así lograr una mejor comprensión del final del Bronce Antiguo III en general y de la ciudad de Jericó en particular. 


\section{Q Bibliografía}

» Albright, W. F. (1961). Abram the Hebrew. A New Archaeological Interpretation, en: Bulletin of the American Schools of Oriental Research 163: 36-54.

»Davis, M. C. (2008). Dame Kathleen Kenyon: Digging up the Holy Land. Walnut Creek: Left Coast Press.

" de Miroschedji, P. (2009). Rise and Collapse in the Southern Levant in the Early Bronze Age, en: Scienze dell'antichità, Storia Archeologia Antropologia 15: 101-129.

" de Miroschedji, P. (2019). Early Bronze Age Palaces in the Southern Levant, en: Bietak, M., Matthiae, P. y Prell, S. (eds.), Ancient Egyptian and Ancient Near Eastern Palaces, Vol. II, Proceedings of a workshop held at the 10th ICAANE in Vienna, 25-26 April 2016. Wiesbaden: Harrassowitz, 159-179.

»Dever, W. G. (1980). New Vistas on the EB IV (MB I) Horizon in Syria-Palestine, en: Bulletin of the American Schools of Oriental Research 237: 35-64.

»Dever, W. G. (1992). Pastoralism and the End of Urban Early Bronze Age in Palestine, en: Bar-Yosef, O. y Khazanov, A. (eds.), Pastoralism in the Levant. Archaeological Materials in Anthropological Perspectives (Monographs in World Archaeology 10). Madison: Prehistory Press, 83-92.

»Di Bennardis/De Bernardi, C. (2014). Ignace Gelb: metodología, historia social y rescate de la diversidad sociocultural en Mesopotamia entre el III y II milenios a.C., en: Claroscuro 13: 40-63.

" Gaastra, J., Greenfield, T. y Greenfield, H. (2020). There and Back Again: A Zooarchaeological Perspective on Early and Middle Bronze Age Urbanism in the Southern Levant, en: PLoS ONE 15(3): e0227255. https://doi.org/10.1371/ journal.pone.0227255

» Gallo, E. (2013). The EB III Fortifications of Tell es-Sultan/Jericho in Area B and B-West, en: Scienze dell'antichità, Storia Archeologia Antropologia 19 (2-3): 49-52.

» Greenberg, R. (2017). No Collapse: Transmutations ofEarlyBronze Age Urbanism in the Southern Levant, en: Höflmayer, F. (ed.), The Late Third Millennium in the Ancient Near East. Chronology, $\mathrm{C}_{14}$, and Climate Change. Chicago: The University of Chicago, 31-58.

"Greenberg, R. (2019). The Archaeology of the Bronze Age Levant. From Urban Origins to the Demise of City-States, 3700-1000 BCE. Cambridge: Cambridge University Press.

» Höflmayer, F. (2017). The Late Third Millennium B.C. in the Ancient Near East and Eastern Mediterranean: A Time of Collapse and Transformation, en: Höflmayer, F. (ed.), The Late Third Millennium in the Ancient Near East. Chronology, C14, and Climate Change. Chicago: The University of Chicago, 1-28.

» Kaniewski, D., Marriner, N., Bretschneider, J., Jans, G., Morhange, C., Cheddadi, R., Otto, T., Luce, F. y van Campo, E. (2019). 300-Year drought Frames Late Bronze Age to Early Iron Age Transition in the Near East: New Palaeoecological Data from Cyprus and Syria, en: Regional Environmental Change. https://doi. org/10.1007/s10113-018-01460-w

» Kaniewski, D., Paulissen, E., Van Campo, E., Al-Maqdissi, M., Bretschneider, J. y 
Van Lerberghe, K. (2008). Middle East Coastal Ecosystem Response to Middleto-Late Holocene Abrupt Climate Changes, en: Proceedings of the National Academy of Sciences 105 (37): 13941-13946.

» Kenyon, K. (1960). Arqueología en Tierra Santa. Barcelona: Ediciones Garriga.

» Kenyon, K. (1966 [1957]). Desenterrando a Jericó. México: Fondo de Cultura Económica.

»Lapp, P. (1966). The Dahr Mirzbaneh Tombs. Three Intermediate Bronze Age Cemeteries in Jordan. New Haven: American Schools of Oriental Research.

" Massa, M. y Palmisano, A. (2018). Change and Continuity in the Long-Distance Exchange Networks between Western/Central Anatolia, Northern Levant and Northern Mesopotamia, c. 3200-1600 BCE, en: Journal of Anthropological Archaeology 49: 65-87.

"Milevski, I. (2016). Intercambio de productos en el Levante meridional durante el Bronce Antiguo. Una perspectiva marxista (Cuadernos de Arqueología Mediterránea 24). Barcelona: Publicaciones del Laboratorio de Arqueología, Universidad Pompeu Fabra de Barcelona.

"Milevski, I. (2010). Centros urbanos y periferias en la Edad del Bronce Antiguo sud-levantina, en: Rivista degli Studi Orientali 83 (1-4): 163-187.

"Nigro, L. (2014). Aside the Spring: Tell Es-Sultan/Ancient Jericho: The Tale of an Early City and Water Control in Ancient Palestine, en: Tvedt, T. y Oestigaard, T. (eds.), A History of Water: Series III, Vol. 1. From Jericho to Cities in the Seas: A History of Urbanization and Water Systems. Londres: I.B. Tauris, 25-51.

» Nigro, L. (2016). Tell es-Sultan 2015. A Pilot Project for Archaeology in Palestine, en: Near Eastern Archaeology 79 (1): 4-17.

»Nigro, L. (2017). The End of the Early Bronze Age in the Southern Levant. Urban Crisis and Collapse Seen from Two zrd Millennium BC-Cities: Tell es-Sultan/ Jericho and Khirbet al-Batrawy, en: Cunningham, T. y Driessen, J. (eds.), Crisis to Collapse. The Archaeology of Social Breakdown. Lovaina la Nueva: Presses Universitaires de Louviain, 149-172.

» Nigro, L. (2019a). Tell es-Sultan/Ancient Jericho in the Early Bronze Age II- III, en: Gallo, E. (ed.), Conceptualizing Urban Experiences. Tell es-Sultan and Tall alHammām Early Bronze cities across the Jordan (ROSAPAT 13). Roma: La Sapienza, 79-108.

"Nigro, L. (2019b). Jericho and the Dead Sea. Life and Resilience, en: Peilstöcker, M. y Wolfram, S. (eds.), Life at the Dead Sea. Proceedings of the International Conference held at the State Museum of Archaeology Chemnitz (smac), February 21-24, 2018, Chemnitz. Ägypten und Altes Testament 96. Münster: Zaphon, 139156.

»Nigro, L. (2020). The Italian-Palestinian Expedition to Tell es-Sultan, Ancient Jericho (1997-2015): Archaeology and Valorisation of Material and Immaterial Heritage, en: Sparks, R. T., Finlayson, B., Wagemakers, B. y Briffa, J. M. (eds.), Digging Up Jericho. Past, Present and Future. Oxford: Archaeopress Archaeology.

» Nigro, L. (2021). Teapot or Milkpot? About the Content of a Small, Spouted Jar from EB IV (2300-2000 B.C.E.) Tell es-Sultan, Ancient Jericho, en: Mediterranean Archaeology and Archaeometry 21 (1): 281-290.

"Nigro, L., Calcagnile, L., Yasin, J., Gallo, E. y Quarta, G. (2019). Jericho and the Chronology of Palestine in the Early Bronze Age: A Radiometric Re-Assessment, 
en: Radiocarbon 61 (1): 211-241.

"Nigro, L., Montanari, D., Mura, F. y Caminiti, R. (2018). Not Invasive Analyses on a Tin-Bronze Dagger from Jericho: A Case Study, en: Mediterranean Archaeology and Archaeometry 18 (1): 199-207.

»Riehl, S. (2008). Climate and Agriculture in the Ancient Near East: A Synthesis of the Archaeobotanical and Stable Carbon Isotope Evidence, en: Vegetation History and Archaeobotany 17: S43-S51.

»Sala, M. (2012). Egyptian and Egyptianizing Objects from EB I-III Tell es-Sultan/ Ancient Jericho, en: Vicino Oriente 16: 275-302.

"Schloen, D. J. (2017). Economic and Political Implications of Raising the Date for the Disappearance of Walled Towns in the Early Bronze Age Southern Levant, en: Höflmayer, F. (ed.), The Late Third Millennium in the Ancient Near East. Chronology, $\mathrm{C}_{14}$, and Climate Change. Chicago: The University of Chicago, 59-71.

»Schwartz, G. M. (2017). Western Syria and the Third - to Second - Millennium B.C. Transition, en: Höflmayer, F. (ed.), The Late Third Millennium in the Ancient Near East. Chronology, C14, and Climate Change. Chicago: The University of Chicago, 87-128.

»Vega Ruiz, I. (2019). Mardikh IIB1 y el archivo C, un hito histórico-arqueológico, en: Arqueología y Territorio 16: 81-95.

"Weiss, H., Courty, M.-A., Wetterstrom, W., Guichard, F., Senior, L., Meadow, R. y Curnow, A. (1993). The Genesis and Collapse of Third Millennium North Mesopotamian Civilization, en: Science 261/5124: 995-1004. 
